\title{
An Efficient Program to Design Linear Accelerator Structural Shielding
}

\author{
M. Sayed \\ Radiation Engineering Department, \\ Egyptian Atomic Energy Authority, EAEA, \\ 3 Ahmed El-Zomar Str., 8th District, Nasr City, Cairo Egypt
}

\begin{abstract}
Structural shield calculations for the radiotherapy treatment room are important to get the requirements of radiation protection. These calculations must perform to get the optimum thickness of treatment room walls in the design step. A Matlab program is proposed to design the structural shield for the linear accelerator. The proposed program based on the national council on radiation protection and Measurements (NCRP) report number 151. The proposed program is constructed from four interfaces to compute the different barriers in the treatment room. Firstly, the design of the treatment room is loaded to the program. Then, the primary and secondary barriers are calculated. Finally, the dose rate at the maze is calculated to select the suitable door design. The proposed program was validated based on recalculate all examples of the NCRP report No 151. Then, we compare the results precisely. The proposed program is simple, and fast.
\end{abstract}

\section{Keywords}

Radiation protection - Linear accelerator - structure shield design

\section{INTRODUCTION}

Structural shielding design aims to limit radiation exposures to an acceptable level. Where, exposure to ionizing radiation by over doses caused cancer. The risk of causing cancer is reduced proportionally by reducing the exposure dose. Consequently, the effective dose from the radiation source (as linear accelerator in this work) to a point outside the treatment room must be reduced using a proper shielding to achieve the protection requirements.

This shielding attenuate the amount of ionizing radiation using a barrier material such as concrete, lead, or steel [1]. The thickness and type of the barrier depends on the radiation type.

Two types of radiation are consider in the radiotherapy applications; primary and secondary radiations. The primary radiation consists of the radiation that emitted directly from the radiation source (as linear accelerator) to any point in the treatment room. On the other hand, secondary radiation is the radiation scattered from interactions with any object in the treatment room. A primary barrier is a structure that used to attenuate the directed beam and any secondary radiation. However, the secondary barrier attenuates the secondary radiation to the appropriate shielding design goal.

The medical centers (or the other radiation facilities) is divided into two areas, controlled area and uncontrolled areas. Controlled area is a limited-access area such as control consoles and treatment rooms. Working conditions in this area are controlled for radiation protection purposes. Radiation dose of the workers in these areas usually individually monitored. On the other hand, uncontrolled areas are all other areas in the surrounding environs. The proper shielding reduces the ionizing radiation to the accepted dose. The effective equivalent dose are $100 \mu \mathrm{Sv} /$ week for controlled areas and $20 \mu \mathrm{Sv} /$ week for uncontrolled areas $[2,3]$. The optimal design of the structural shielding must be determined precisely before beginning construction. Where, any correction after the facilities completed is expensive.

However, in the facility design, possible future needs should be take into consideration for replacing the equipment with higher radiation energies. There are many calculations for structural shield such as primary and secondary barrier and maze calculations. The proposed program can be used to achieve this goal easily and fast. Furthermore, the program can be applied on any structural shield design of the treatment room.

The proposed work introduces a Matlab program to calculate a structural shielding of the radiotherapy treatment room. The treatment room design is loaded to the proposed program. Subsequently, the proper shield for this design is calculated based on the NCRP report no. 151 [3].

\section{MATERIAL AND METHOD}

The calculation method in the proposed work evaluates thicknesses of the barriers for a radiotherapy treatment room based on the tenth-value layer (TVL) concept as in [3] . TVL is the thickness of the material that is used in the structural shield to absorb $90 \%$ of radiation intensity. The material of the structural shield could be steel, lead, ordinary concrete or any other shielding material. In the proposed program, we insert the parameters of these materials in the database and user can select the desired material from a popup menu as shown in the results section.

There are several parameters must be defined in the design for public and workers in the radiotherapy center. Such as use factor (U), weekly workload (W), shielding design goal (P) and occupancy factor (T). $U$ is the fraction of a primary-beam workload that is directed in the direction of a given primary barrier. $W$ is the time integral of the absorbed-dose rate computed at the depth of the maximum absorbed dose away from the radiation source by a one meter. Unit of the workload is Gy /week. $P$ determines the accepted level of radiation at the desired location, which depends on the class of person and the class of area where the person is located. $P$ is measured in $\mathrm{Sv} /$ week. $T$ is the average fraction of time that the maximally exposed individual is present while the beam is on. $T$ is defined for each area. For example, the value of $T$ for the outdoor areas equals to $1 / 40$. Hence, The values of $\mathrm{W}, \mathrm{T}$, and $\mathrm{F}$ are detrmined for the desired design goal. Then, the barriers thicknesses are 
computed to achieve the value of $(\mathrm{P})$ for the selected area.

The main steps for structural shielding calculations of the radiotherapy treatment room are shown in block diagram of figure 1. The design of the treatment room is loaded to the program. Then, the thicknesses of barriers are calculated. The dose rate at the maze due to the transmitted and scatting radiation is calculated. In the following sections, calculations of the primary thickness and other terms of the structural shielding are discussed.

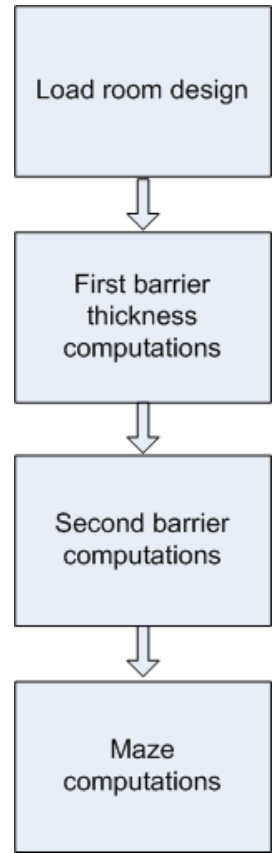

Figure 1: The main steps for shielding calculation of the radiotherapy treatment room

\subsection{Primary barriers}

Primary barrier is the barrier in which the primary beam of the radiation source (such as beam of linear accelerator) falls on it. The transmission factor (B) of the barrier reduces the radiation field to an acceptable level. The dose limit for a non-controlled area is less than $.02 \mathrm{mSv} /$ week [4]. Equation (1) that represents this factor as in [3]:

$\mathrm{B}=\frac{\mathrm{Pd}_{\mathrm{pri}}}{\mathrm{WUT}}$

$d_{\text {pri }}$ is the distance from the isocentre to outside of the barrier

The number of the tenth-value layers (TVLs) required to get (B) is determined from equation [3]:

No. of TVLs $=\log _{10}\left(\frac{1}{B}\right)$

The thickness of the primary shield (tpri) is calculated as equation (3)

tpri $=$ TVL1 $+($ No. of TVLs -1$)($ TVLe $)$

Where, TVL1 and TVLe are the first and equilibrium tenth-value layers (meters) of the primary radiation. The vales of these TVLs are stored in the database of the program at the different energies for the all-shielding materials [3]. For example, if the energy of the recommended linear accelerator is $6 \mathrm{Mev}$ the first and tenth-value layer are illustrated in the table 1. The densities of the shielding materials also considered as represented in this table

Table 1 the primary-barrier TVLs for $6 \mathrm{MV}$ according to the shielding material [3]

\begin{tabular}{llll}
\hline material & $\begin{array}{l}\text { Density } \\
(\mathrm{g} / \mathrm{cm} 3)\end{array}$ & TVL1 $(\mathrm{cm})$ & $\begin{array}{l}\text { TVLe } \\
(\mathrm{cm})\end{array}$ \\
\hline $\begin{array}{l}\text { Ordinary } \\
\text { Concrete }\end{array}$ & 2.35 & 37 & 33 \\
Steel & 7.87 & 10 & 10 \\
lead & 11.35 & 5.7 & 5.7 \\
\hline
\end{tabular}

Then, the expected dose-equivalent rate per hour (IDR) that is calculated in Sv/hr according to equation (4):

$I D R=\frac{D_{0} B}{d_{p r i}^{2}}$

Where, $\mathrm{D}_{0}$ is the maximum absorbed-dose output rate at the isocentre. After that, the weekly averaged dose equivalent rate (WADR) [Sv/week] is calculated as in equation (5)

$W A D R=\frac{I D R W U}{D_{0}}$

Finally, the dose equivalent in-any-one-hour $(\mathrm{Rh})[\mathrm{Sv} / \mathrm{hr}]$ is calculated based on equation (6).

$R_{h}=\frac{M}{x} * W A D R$

Where

$\mathrm{x}$ is the number of working hour in a week

$\mathrm{M}$ is the ratio of the maximum number of patients in an hour to the average number of patients in an hour.

The value of the $\mathrm{R}_{\mathrm{h}}$ must satisfy the shielding design goal (P). For example the value of $\mathrm{P}$ for the uncontrolled area is equal to $20 \mu \mathrm{Sv}$ in any-one-hour. If the calculated value of $\mathrm{R}_{\mathrm{h}}$ is greater than $20 \mu \mathrm{Sv}$, At least one $H V L$ at the operating energy of the linear accelerator must be added to the $t$ pri. However, for more safety two values of HVL should be added to the tpri [3]. The value of $R_{h}$ is tested using the proposed program and a suitable number from HVL are added to satisfy the design goal. Therefore, the user of the proposed program needn't to be an expert in radiation protection, where the requirements of this protection are satisfied by the program automatically.

\subsection{Secondary barrier}

Secondary barrier is designed to attenuate the leakage radiation from the treatment room walls and the treatment head and the scattered radiation from the patient [4].

\subsubsection{Leakage radiation}

The transmission factor $\left(\mathrm{B}_{\mathrm{L}}\right)$ for the Leakage radiation is as follows:

$\mathrm{B}_{\mathrm{L}}=\frac{\mathrm{Pd}_{\mathrm{L}}^{2}}{10^{-3} \mathrm{WT}}$

Where, $d_{L}$ is the distance from the point of interest to the isocentre in meter. The factor $10^{-3}$ arises from the assumption that leakage radiation from the accelerator head is $0.1 \%$ of the useful beam. $\mathrm{B}_{\mathrm{L}}$ depends on the values of $\mathrm{P}, \mathrm{W}$, and $\mathrm{T}$ as in the equation (1). The dose equivalent $\left(\mathrm{H}_{\mathrm{L}}\right)$ [Sv/week] is computed as in equation (8) 
$H_{L}=W T d_{L}^{-2} \times 10^{-3} \times 10^{-\left[1+\frac{t_{p r i}+T V L}{T V L e}\right]}$

\subsubsection{Scattered radiation}

The barrier transmission factor (Bp) that required shielding against radiation scattered by the patient is defined as follows:

$B_{p}=\frac{p d_{\text {sca }}^{2} d_{\text {sec }}^{2}}{a W T\left(\frac{F}{400}\right)}$

Where

dsca is the distances from the patient to the radiation source dsec is the distances from the patient to the point of interest a scatter fraction defined at the dsca.

$\mathbf{F}$ field area at mid-depth of the patient at one meter

The value 400 assumes the scatter fractions are normalized to those measured for a field size of dimensions $20 \mathrm{~cm} \times 20 \mathrm{~cm}$.

Radiation scattered by a patient is generally less than $0.1 \%$ of the incident radiation per $0.1 \mathrm{~m}^{2}$ area irradiated [3]. The photons scattered by the patient and by the wall are nearly the same energy. Required thickness of shield from both of patient scatter and wall scatter are compared. Then the largest one is taken and one-half value layer (HVL) is added $[2,5]$.

\subsubsection{Maze}

Different radiations types reach the maze door due to the scattering photons from patient and the room surfaces. Moreover, the photons of head-leakage radiation pass through the inner maze walls. The equations that calculate these types of radiation are represented in report 151 . Besides radiation protection from these radiation types, the maze door should be designed as light as possible to move it easily. Figure (3) shows the parameters that are used to calculate total radiation at maze door.

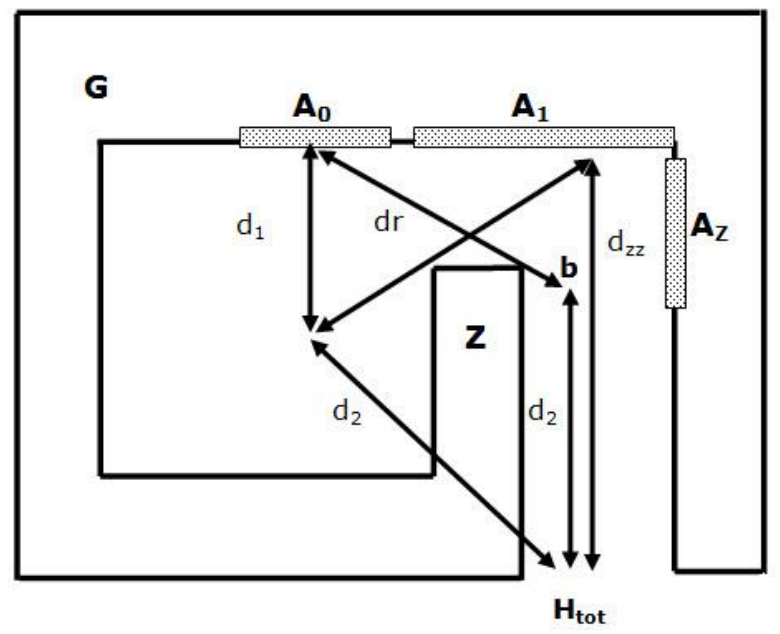

Figure 2: Input parameters to calculate total radiation at the $\mathrm{G}$ and $\mathrm{Z}$ represent the wall index maze door [3]

$\mathrm{A}_{0}$ beam area at the first scattering surface,

$\mathrm{A}_{\mathrm{z}}$ represents the cross-sectional area of maze inner entry,

$\mathrm{d}_{\mathrm{h}}$ perpendicular distance from the first reflection surface to the target,

$\mathrm{dr}$ distance from beam center at the first reflection to Point $b$,

$\mathrm{dz}$ centerline distance along the maze from the maze door to Point b
The radiation at the maze is arising from head-leakage radiation and scattering of photons from the room surfaces. There are four types of radiation components at the maze [3]:

Dose equivalent per week due to both of scatter of the primary beam from the room surfaces $(H S)$, head-leakage photons scattered by the room surfaces (HLS), primary beam scattered from the patient $(\mathrm{Hps})$, and leakage radiation which is transmitted through the inner maze wall (HLT).

Equations 10 up to 13 represent the value of each radiation components

$$
H_{s}=\frac{W U_{G} \alpha_{0} A_{0} \alpha_{z} A_{z}}{\left(d_{h} d_{r} d_{z}\right)^{2}}
$$

$\boldsymbol{\alpha}_{0}$ reflection coefficient at the first scattering surface $A_{0}$ $\boldsymbol{A}_{\mathbf{0}}$ beam area at the first scattering surface (m2)

$\boldsymbol{\alpha}_{\mathbf{z}}$ reflection coefficient for second reflection from the maze surface $A z$

$\boldsymbol{A}_{\mathbf{Z}}$ cross-sectional area of maze inner entry projected onto the maze wall from the perspective of the irradiated primary barrier $A 0(\mathrm{~m} 2)$

$\boldsymbol{d}_{\mathbf{h}}$ perpendicular distance from the target to the first reflection surface (meters)

$\boldsymbol{d}_{\mathbf{r}}$ distance from beam center at the first reflection, past the edge of the inner maze wall, to point $b$ on the midline of the maze (meters)

$\boldsymbol{d}_{\mathbf{z}}$ centerline distance along the maze from Point $\mathrm{b}$ to the maze door (meters)

$$
H_{L S}=\frac{L_{f} W_{L} U_{G} \alpha_{1} A_{1}}{d_{\mathrm{sec}} d_{z z}}
$$

$\boldsymbol{L}_{\mathbf{f}}$ head-leakage radiation ratio at $1 \mathrm{~m}$ from the target $\boldsymbol{W}_{\mathbf{L}}$ workload for leakage radiation (Gy week-1)

$\alpha_{1}$ reflection coefficient for scatter of leakage radiation from Wall G

$A_{1}$ area of Wall G that can be seen from the maze door (m2)

$\boldsymbol{d}_{\mathbf{z z}}$ centerline distance along the maze (meters)

$$
H_{p S}=\frac{a(\theta) W U_{G}\left(\frac{F}{400}\right) \alpha_{1} A_{1}}{\left(d_{\mathrm{sec}} d_{\mathrm{sc} a} d_{z z}\right)^{2}}
$$

$\boldsymbol{a}(\boldsymbol{\theta})$ scatter fraction for patient-scattered radiation at angle $\theta$ (this value is inserted the program database)

$\boldsymbol{F}$ field area at mid-depth of the patient at one meter $(\mathrm{cm} 2)$

$$
H_{L T}=\frac{L_{f} W_{L} U_{G} B}{d_{L}^{2}}
$$

$\boldsymbol{B}$ transmission factor for Wall $\mathrm{Z}$ along the oblique path traced by $d \mathrm{~L}$

$\boldsymbol{d} \mathbf{L}=$ distance from the target to the center of the maze door through the inner maze wall (meters).

Each previous component of radiation is calculated. Then the total dose equivalent at the maze $\left(\mathrm{H}_{\mathrm{G}}\right)$ with the beam aimed at Wall $\mathrm{G}$ is computed according equation (14):

$$
H_{G}=f H_{s}+H_{L S}+H_{p s}+H_{L T}
$$


$f$ represents the fraction of the beam transmitted through the patient. $\mathrm{f}$ is equal to 0.25 for 6 to $10 \mathrm{Mev} \mathrm{x}$-ray [6].

The total dose equivalent $\left(H_{\mathrm{Tot}}\right)$ at the maze door from photon leakage radiation and scattered radiation is estimated as in equation (15) [6]

$$
H_{\text {tot }}=2.64 H_{G}
$$

\section{RESULTS AND DISCUSSION}

All obtained results in this section were compared with the NCRP report No. 151 to validate the proposed program. Calculations steps that represented in figure 1 were computed based on the proposed work. Firstly, the calibration step is performed according to the dimensions of the design to get accurate distances for primary barrier and secondary barrier, etc. Figure (3) represents the loaded design into the proposed Matlab program. This figure is similar to the figure 7.1 in the report 151. We use this figure to validate the proposed program calculations. The scale factor is computed based on a known distance in the loaded figure. For example, to find the drawing scale for the loaded design, the real distance is $4.5 \mathrm{~m}$ and the distance between start and end of line in the loaded design is equal to 182 pixels. Thus, the factor is equal to 40.2. Therefore, any dimension captured from the figure must be divided by the factor (40.4). This step is only a scaling step to convert the dimensions of the loaded design to real.

In the second step, the primary barrier is calculated based on equations 1 , and 2 . Figure 4 represents the interface of the primary barrier calculations. Firstly, area type is selected from the popup menu to define the dose limit. Then, the shielding martial and the energy of the linear accelerator and the occupancy factor are selected. All popup menus in the program are linked to a database that includes the value for each selection. The source of this database is the NCRP report 151 [2].

Subsequently, the remaining input parameters are entered into the text boxes. Finally, the thickness of the primary barrier at the selected location is computed.

After that, the secondary barrier is computed as shown in figure 5. Two types of radiation are considered in this barrier scattered and leakage radiation. The input parameters to calculate this barrier are $\mathrm{W}, \mathrm{U}$, and $\mathrm{T}$ as in the primary barrier calculation. Therefore, users of the program need not to enter these values again. User interface of the secondary barrier includes only the distance and angle of the selected area.

Different dimensions must be concerned to compute the dose equivalent at the door. Figure 2 represents the input dimensions to calculate the maze calculation. Finally, Figure 6 represents the user interface of the maze calculations.

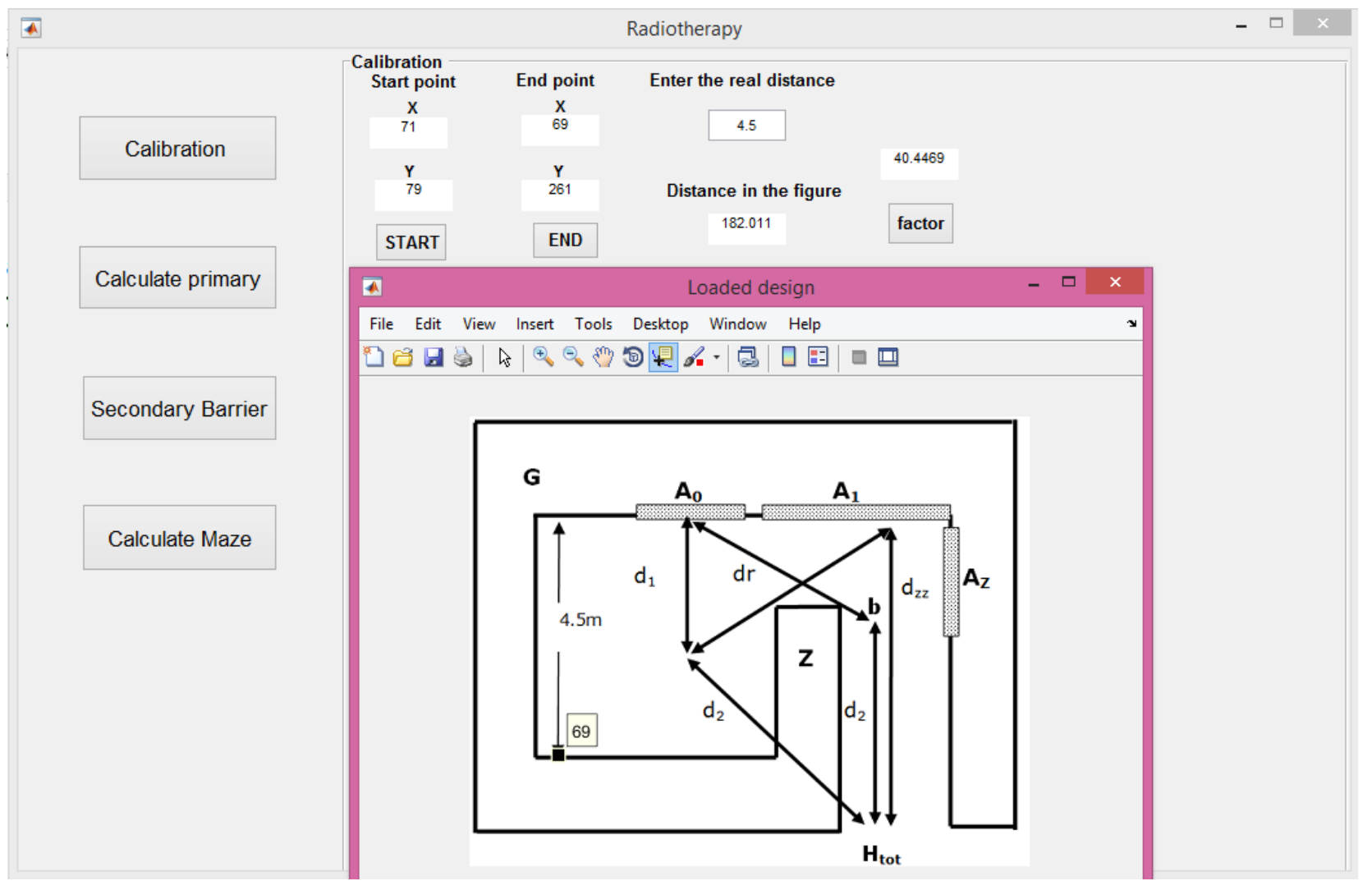

Figure 3 the calibration calculations 


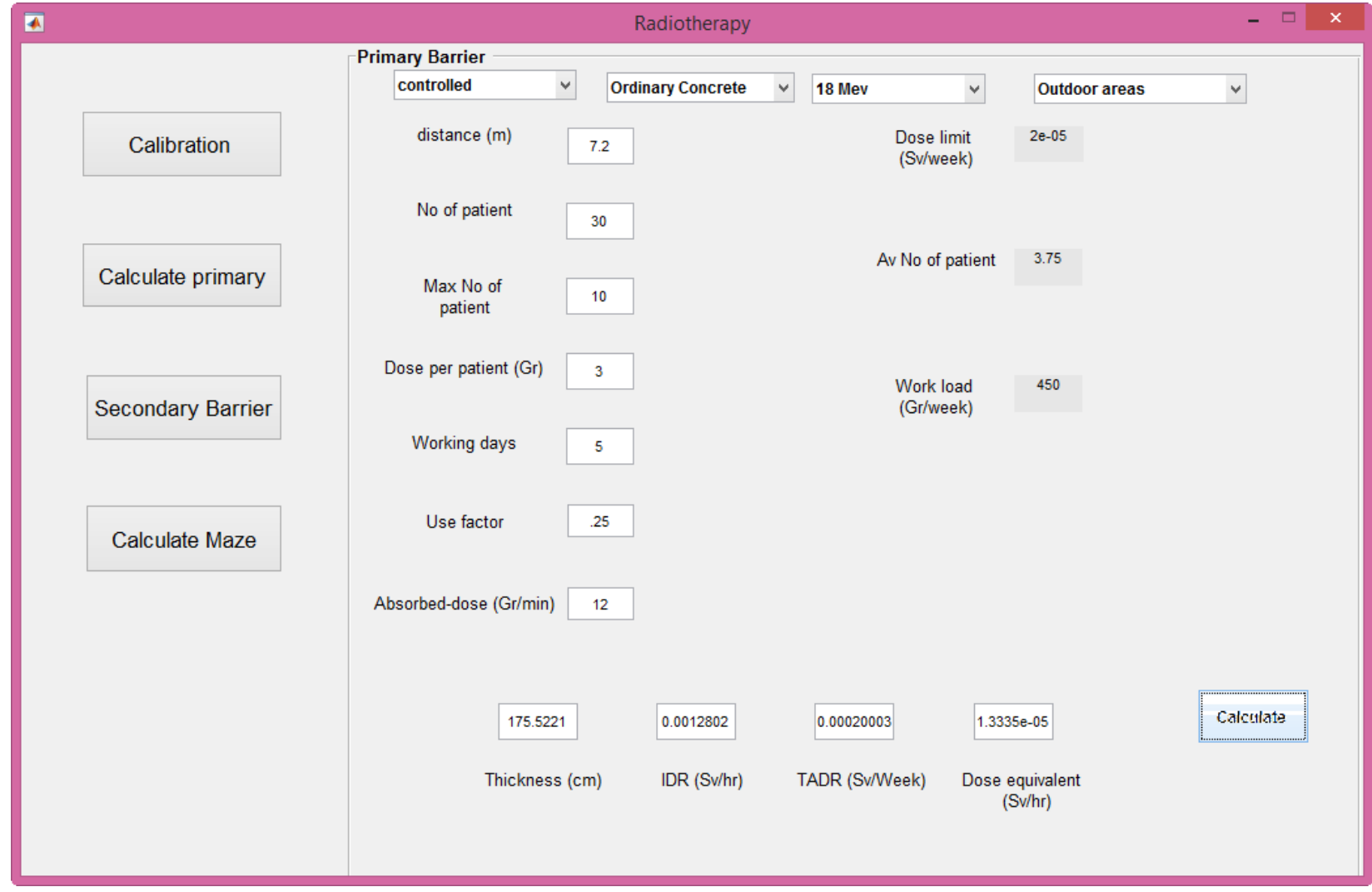

Figure 4 The Primary Barrier Calculations

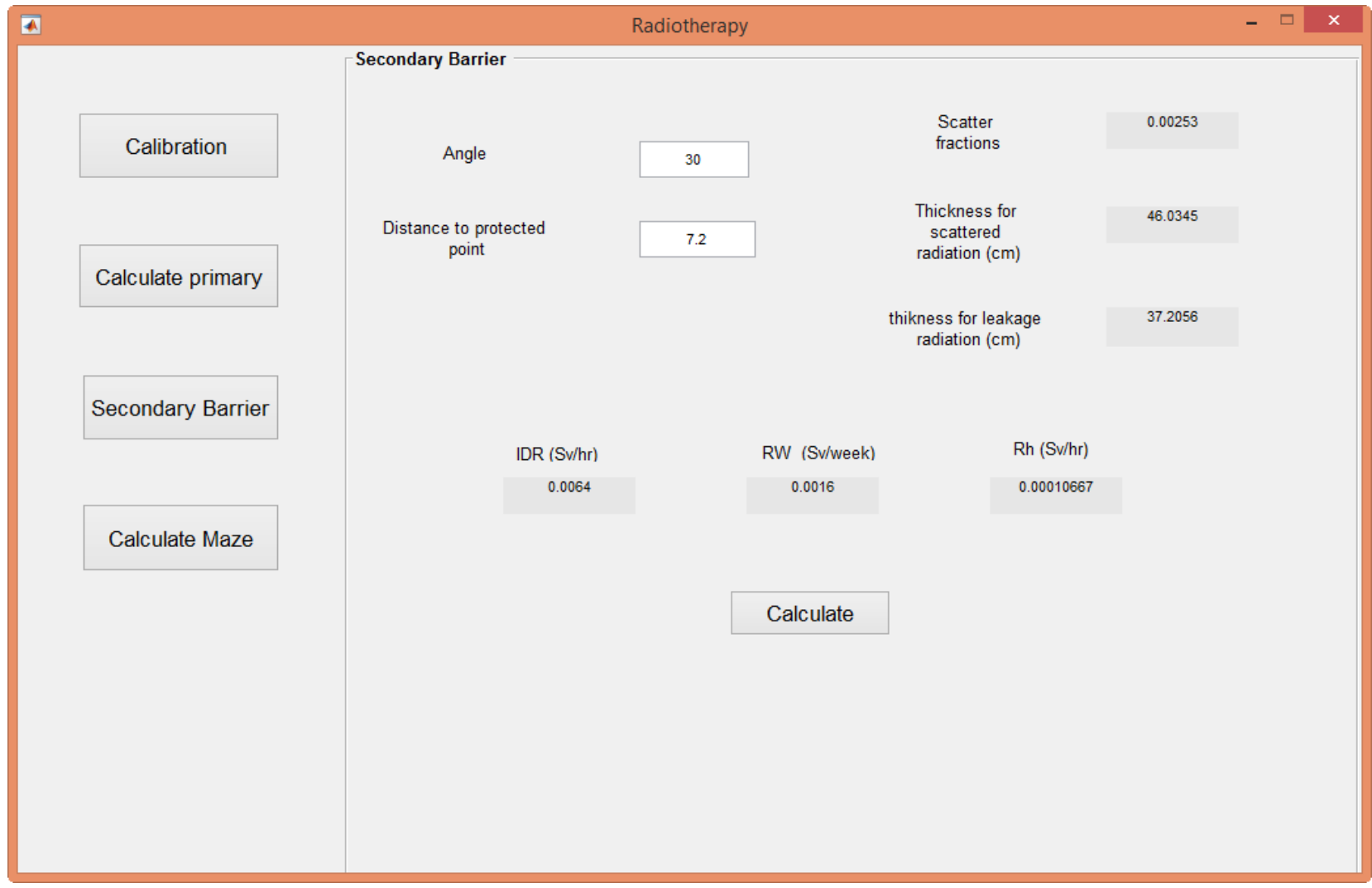

Figure 5 The Secondary Barrier Calculations 


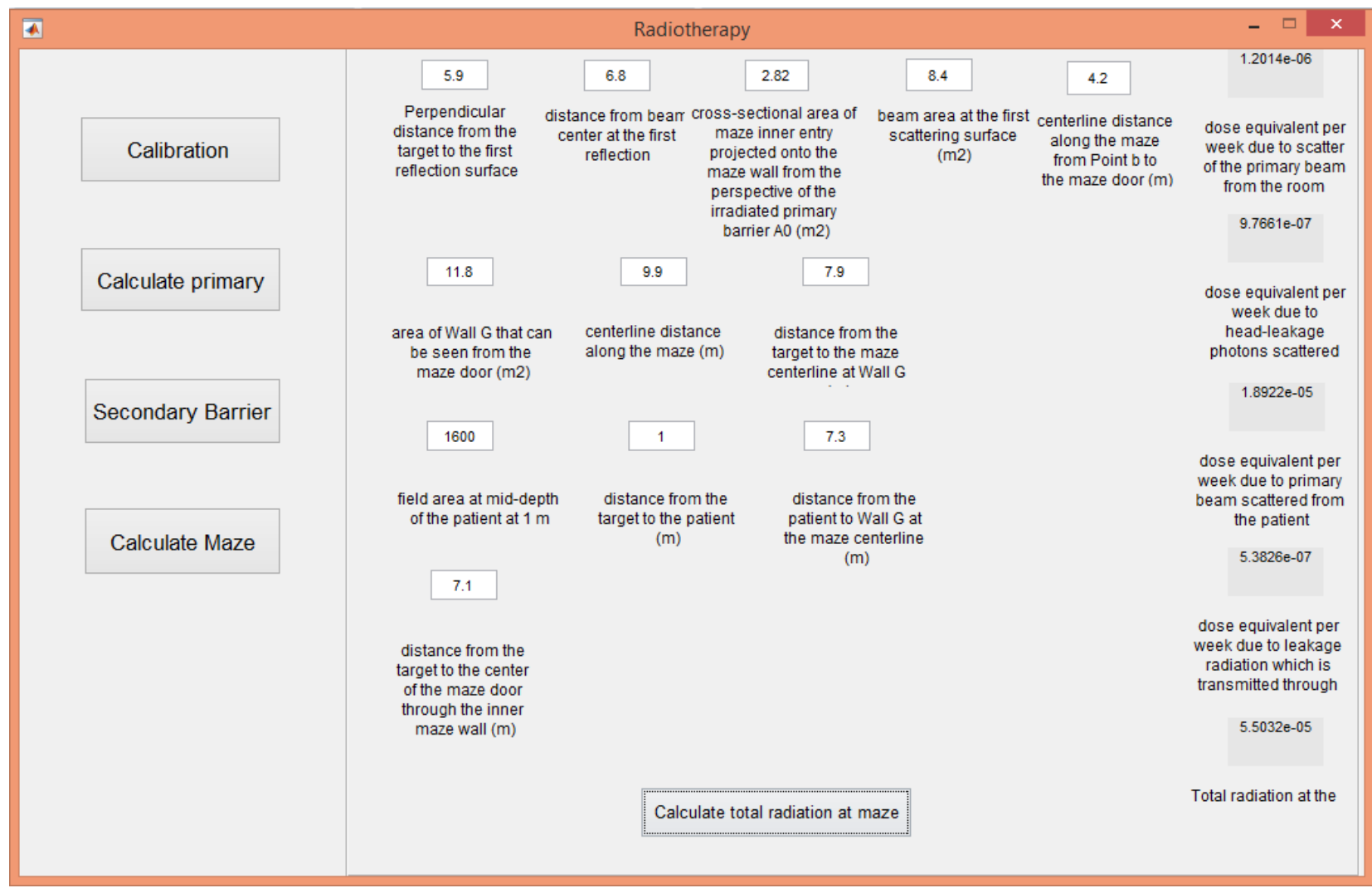

Figure 6 The Maze calculations

Based on the proposed program, the design of the structural shielding for the radiotherapy room can be performed. Besides that, the dose limits can be determined which required by the NCRP easily.

To validate the program we compute the primary and secondary barriers for linear accelerator as example 7 in the report 151 . Table 2 represents comparison between the proposed program and the report calculations. The primary barrier in the table was calculated for controlled area, and $18 \mathrm{Mev}$ linear accelerator as shown in fig 4 . Where, the allowed dose $(\mathrm{Rh})$ is $20 \mu \mathrm{Sv}$. However, the calculated dose according to equations 1 , and 2 for tpri $=149 \mathrm{~cm}$ is equal to $36 \mu \mathrm{Sv}$. Thus, two HVL were added to $\mathbf{t}_{\text {pri. }}$. The final value for primary thickness is equal to $176 \mathrm{~cm}$ and the dose for this thickness was $9 \mu \mathrm{Sv}$ that is lower than 20 $\mu \mathrm{Sv}$. As mentioned before inexperienced user in the radiation protection can use the proposed program to find the thickness for the structural shield for the linear accelerator. Where the previous step is performed automatically in the program.

Table 2 comparison between the proposed program and the report 151

\begin{tabular}{|c|c|c|}
\hline Parameter & $\begin{array}{c}\text { Proposed } \\
\text { program }\end{array}$ & Report 151 \\
\hline \multicolumn{3}{|c|}{ Primary barrier } \\
\hline IDR(Svh $^{-1)}$ & $5.1 \mathrm{e}-3$ & $5.1 \mathrm{e}-3$ \\
\hline $\mathbf{R}_{\mathbf{W}}(\mathbf{S v})$ & $800 \mathrm{e}-6$ & $800 \mathrm{e}-6$ \\
\hline $\mathbf{R}_{\mathbf{h}}(\boldsymbol{\mu} \mathbf{S v})$ & 35.8 & 36 \\
\hline $\mathbf{t}_{\text {pri }}$ & 175.5 & 176 \\
\hline \multicolumn{3}{|c|}{ Secondary barrier } \\
\hline $\begin{array}{c}\text { Thickness for } \\
\text { scattered radiation }\end{array}$ & 37.2 & 37.3 \\
\hline $\begin{array}{c}\text { Thickness for } \\
\text { leakage radiation }\end{array}$ & 46.03 & 46.1 \\
\hline \multicolumn{2}{|c|}{ Maze Door } \\
\hline
\end{tabular}

\begin{tabular}{|c|c|c|}
\hline $\mathbf{H}_{\mathbf{s}}(\boldsymbol{\mu S \mathbf { S v } )}$ & 1.2 & 1.2 \\
\hline $\mathbf{H}_{\mathbf{L s}}(\boldsymbol{\mu S v})$ & 0.977 & 0.970 \\
\hline $\mathbf{H}_{\mathbf{p s}}(\boldsymbol{\mu} \mathbf{S v})$ & 18.9 & 18.85 \\
\hline $\mathbf{H}_{\mathbf{L T}}(\boldsymbol{\mu S v})$ & 0.538 & 0.536 \\
\hline $\mathbf{H}_{\text {tot }}(\boldsymbol{\mu S \mathbf { S v }})$ & 55.03 & $55 . .02$ \\
\hline
\end{tabular}

As illustrated in the previous table, the results are quite similar. Therefore, the proposed program can be used to determine the thickness of the structural shielding of the radiotherapy room based on this report 151 .

As a case study based on the proposed program, the distance from the source of radiation to the primary barrier is varied from, $5 \mathrm{~m}$ up to $10 \mathrm{~m}$ and the thickness according to these distances are calculated according to the required dose limits. Figure 7 illustrates the values for the calculated thickness according to the selected distance. The relation between any parameters can be studied according to the proposed program.

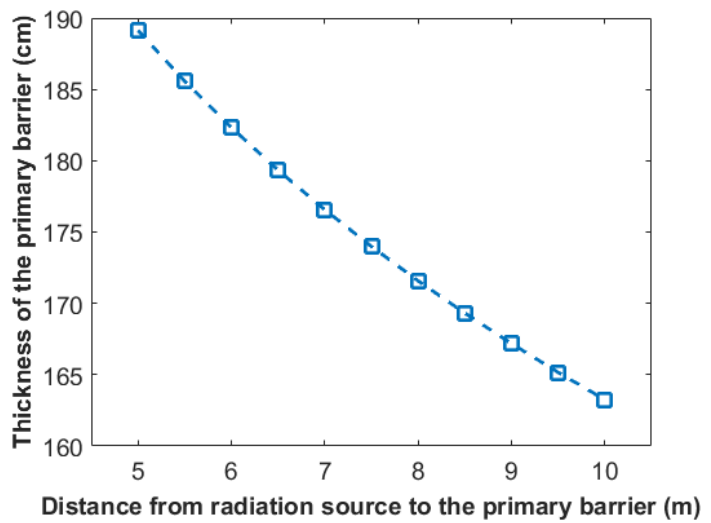

Figure 7 study of the primary thickness as a function of the distance from source of radiation 


\section{CONCLUSION}

The proposed computer program is designed to calculate the structure shield parameters of radiotherapy linear accelerator. The proposed program has four interfaces to determine the thickness of primary and secondary barriers. Moreover, the shielding barrier for maze door can be calculated. The interface of the program is easy-to-use. User selects the suitable shield martial from predefined several saved materials for certain energy linear accelerator such as lead, or ordinary concrete. The proposed program can be used to get a suitable design of each part of structural shield beside the requirements of the radiation protection. Based on the proposed program, the radiation doses at different area around the treatment room can be determined based on the 151 report. The proposed program is easy to use. The user can upload any design for the structural shield of radiotherapy treatment room and get the suitable thickness for the primary and secondary parries.

\section{REFERENCES}

[1] S. Jayaraman and L. H. Lanzl, Clinical radiotherapy physics: Springer Science \& Business Media, 2011.

[2] I. A. E. Agency, Radiation protection in the design of radiotherapy facilities: Internat. Atomic Energy Agency, 2006.

[3] N. Rohrig, "Structural Shielding Design and Evaluation for Megavoltage X-and Gamma-Ray Radiotherapy Facilities, NCRP Report No. 151," ed: LWW, 2006.

[4] N. C. o. R. Protection and Measurements, "Recent applications of the NCRP public dose limit recommendation for ionizing radiation," ed: NCRP Washington, DC, 2004.

[5] K. H. Darby, T. C. Mcclanaha, A. Moore, C. T. Crutcher, and M. A. Megonigal, "Accelerator Facility Shielding Design," 2015.

[6] P. McGinley and J. James, "Maze design methods for 6and $\quad 10-\mathrm{MeV}$ Accelerators," Radiation Protection Management, vol. 14, pp. 59-64, 1997. 